\title{
Nutritional Literacy Among Uninsured Patients With Diabetes Mellitus: A Free Clinic Study
}

Bianshly Rivera Rivero ${ }^{1}$, Alena Makarova ${ }^{2}$, Dina Sidig ${ }^{3}$, Saniya Niazi ${ }^{4}$, Rasha Abddelgader ${ }^{3}$, Sabbir Mirza $^{2}$, Hadi Joud ${ }^{2}$, Mustafa Urfi ${ }^{5}$, Abdillahi Ahmed ${ }^{2}$, Omar Jureyda ${ }^{5}$, Firaas Khan ${ }^{2}$, Justin Swanson ${ }^{6}$, Maqsood Siddique ${ }^{7}$, Natalia Weare-Regales ${ }^{8}$, Abu-Sayeef Mirza ${ }^{9}$

1. Endocrinology, Diabetes and Metabolism, University of South Florida, Tampa, USA 2. Medicine, University of South Florida Morsani College of Medicine, Tampa, USA 3. General Medicine, Red Crescent Clinic, Tampa, USA 4. General Medicine, Red Crescent Clinic, Tampa, USA 5. Miscellaneous, University of South Florida College of Arts and Sciences, Tampa, USA 6. Miscellaneous, University of South Florida College of Public Health, Tampa, USA 7. Cardiology, James A. Hailey Veterans Affairs Medical Center, Tampa, USA 8. Endocrinology, Diabetes and Metabolism, James A. Hailey Veterans Affairs Medical Center, Tampa, USA 9. Internal Medicine, University of South Florida Morsani College of Medicine, Florida, USA

Corresponding author: Bianshly Rivera Rivero, bianshly@usf.edu

\section{Abstract \\ Objective}

Evaluate nutrition literacy in uninsured subjects with diabetes mellitus (DM) who presented to free diabetes management classes.

\section{Design}

This single-site, cross-sectional observational study recruited thirty subjects from a free clinic for uninsured patients to attend diabetes mellitus, self-management classes. Before starting the classes, DM care-related data were collected, and subjects were administered the Nutrition Literacy Assessment Instrument (NLit). The assessment covers six subscales in nutrition and categorizes results into three possible categories: the likelihood of poor nutrition literacy (NLit Score $\leqslant 44$ ), the possibility of poor nutrition literacy (NLit Score$45-57$ ), and the likelihood of good nutrition literacy (NLit score $\geqslant 58$ ).

\section{Results}

Median glycated haemoglobin (HbA1c) was 7.45\% for study participants. The mean NLit score was 38.1 (SD \pm 9.4), correlating with a likelihood of poor nutrition literacy. All participants had either likelihood or the possibility of poor nutrition literacy based on the NLit Assessment. There were no participants who scored in the range of likelihood of good nutrition literacy. Subjects who scored in the range of likelihood of poor nutrition literacy had a significantly higher mean HbA1c (8.6 \%) than those who scored in the possibility of poor nutrition literacy $(6.9 \%, \mathrm{p}=0.005)$.

Received 02/26/2021

Review began $03 / 26 / 2021$ Review ended 06/15/2021 Published 07/13/2021

\section{() Copyright 2021}

Rivera Rivero et al. This is an open access article distributed under the terms of the Creative Commons Attribution License CC-BY 4.0., which permits unrestricted use, distribution, and reproduction in any medium, provided the original author and source are credited.

\section{Conclusions}

Poor nutrition literacy is associated with worse glycemic control among uninsured subjects with diabetes mellitus.

Categories: Endocrinology/Diabetes/Metabolism, Epidemiology/Public Health

Keywords: diabetes mellitus, nutrition literacy, health literacy, nutrition, free clinics, uninsured

\section{Introduction}

As of 2018, the Center for Disease Control and Prevention reported 34.2 million Americans with DM and 88 million with pre-diabetes. One out of ten Americans has diabetes, and one-third are at a high risk of developing diabetes. Furthermore, diabetes management issues have a direct impact on the health and socioeconomic wellbeing of our society. In 2017, the US's estimated total diabetes direct and indirect cost was $\$ 327$ billion [1]. For individual patients, the cost burden of diabetes can be devastating. Patients with diabetes spend 2.3 times more on medical expenditures than non-diabetics [2]. Unfortunately, diabetes is also more prevalent in lower-income, minority groups, and the medically uninsured $[3,4]$. This latter group has been directly associated with poor diabetes outcomes [3,5]. Targeting behavioural factors and disease knowledge could lessen hyperglycemia, potentially reducing the morbidity, mortality, and cost associated with this disease among the uninsured, a poorly represented demographic in the medical literature.

Health literacy is a measure of a person's ability to obtain, read, understand, and use health information to make appropriate health decisions and to follow treatment recommendations [6]. Previous studies have shown that low health literacy is associated with poor glycemic control, poor diabetes knowledge, fewer selfmanagement behaviours, and higher healthcare costs [7-11]. Furthermore, factors such as living in poverty, 
low education level, being part of a minority group, and lack of health insurance have all been linked to low health literacy [12]. The National Institutes of Health, Healthy People 2020, and the Institute of Medicine have identified poor health literacy to be a barrier to health. According to The US Office of Disease Prevention and Health Promotion, improvements in health practice that address low health literacy are needed to reduce disparities in health status [13].

An essential component of health literacy is nutrition literacy, defined as how individuals can obtain, process, and understand nutrition information [14,15]. It also measures the skills necessary to make appropriate nutritional decisions [16]. Our study measured nutrition literacy in a group of uninsured patients with diabetes who presented to a free educational diabetes self-management class. We hypothesized that uninsured patients with diabetes would have the likelihood or possibility of poor nutrition literacy, as previously published research reported low health literacy in patients who were uninsured and had lower socioeconomic status.

\section{Materials And Methods}

This single-site, cross-sectional observational pilot study describes the baseline characteristics, diabetes care-related data, and nutritional literacy of uninsured patients attending a monthly diabetes education class at a free clinic in Tampa, Florida, between October 2018 to March 2019. Uninsured men and women aged 18 years or older who had a diagnosis of DM were invited to participate in the study. Exclusion criteria included: insured subjects, pediatric subjects, and pregnant women. This free diabetes self-management class was publicly advertised as a University-funded resource that awarded voluntary participation with free supplies that included glucometers, test strips, and lancets. Subjects with diabetes, evaluated and managed at the free clinic, were encouraged and referred to attend this class.

This study was approved by the University of South Florida Institutional Review Board with an expedited review (protocol \#Pro00023920). Each patient was provided written informed consent to have recorded deidentified data collected via surveys utilizing portable electronic devices.

We gathered self-reported diabetes-care related data and the NLit Assessment answers. The NLit Assessment Instrument is a 64-item survey that has been validated and demonstrated to be reliable at measuring nutrition literacy (entire reliability 0.97; CI- 0.96-0.98 and test-retest reliability 0.88; CI, 0.850.90) [16]. The assessment covers six subscales: Nutrition \& Health; Energy Sources in Food; Household Food Measurements; Food Label \& Numeracy; Food Groups; and Consumer Skills. It categorizes results into three possible categories: the likelihood of poor nutrition literacy (NLit Score $\leqslant 44$ ), the possibility of poor nutrition literacy (NLit Score 45-57), and the likelihood of good nutrition literacy (Nlit score $\geqslant 58$ ) [16].

Data were collected and managed using REDCap electronic data capture tools hosted at the University of South Florida. REDCap (Research Electronic Data Capture) is a secure, web-based application designed to support data capture for research studies, providing our study with an intuitive interface for a validated data entry. All further data analyses were performed using SAS 9.4 (SAS Institute, Cary, NC). Differences in continuous outcomes between groups were tested using Welch's t-test for two groups and Welch's one-way analysis of variance (ANOVA) for three or more groups. A two-tailed value of $p<0.05$ was selected to indicate statistical significance [17].

\section{Results}

\section{Patient characteristics}

This free clinic population has reported chronic disease incidence and socioeconomic factors in prior studies [4,18]. From October 2018 to March 2019, a total of 30 patients consented to participate in the survey before attending a free diabetes education class. The median age was 60 years old $(n=30)$; $50 \%$ were female and 50\% male (Table 1). Their education level ranged widely, from no education to post-graduate education. Most subjects had Type 2 DM (96\%), and 50\% were on oral medications. Subjects expressed that their barriers to diabetes care included the cost of medication (33\%) and the cost of healthcare provider visits (33\%). Only $10 \%$ of patients expressed that lack of education on diabetes treatment was a barrier (Table 1).

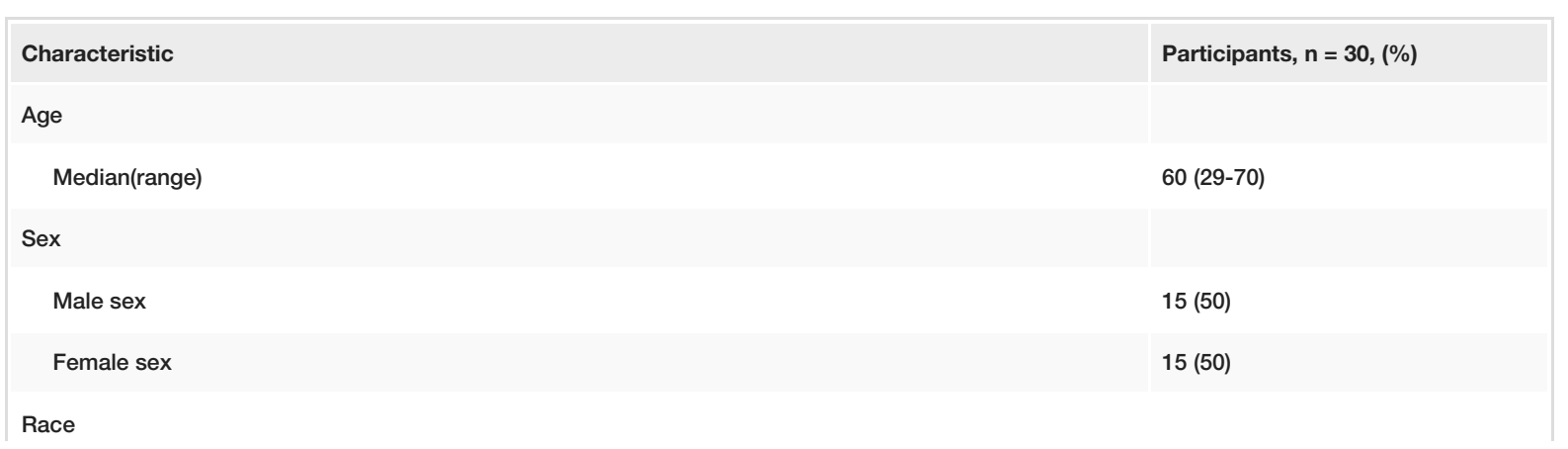




\section{Cureus}

$\begin{array}{lr}\text { Middle Eastern } & 13(43)\end{array}$

Latino / Hispanic $\quad 2(7)$

$\begin{array}{ll}\text { Black } & 6(20)\end{array}$

White $2(7)$

Asian-Indian Subcontinental $\quad 5$ (17)

Other $2(7)$

Education level

None

Elementary

Middle school

$2(7)$

High school

8 (27)

College degree

$12(40)$

Graduate school

Post-graduate

$2(7)$

Employment

Employed

$13(43)$

Unemployed

$17(57)$

Comorbidities

Hypertension

19 (63)

Hyperlipidemia (treated with statin)

$16(53)$

Exercise regimen

Yes

$22(73)$

No

8 (27)

Diabetes type

Type 1

1 (3)

Type 2

29 (97)

Diabetes medication regimen

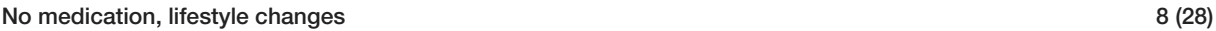

$\begin{array}{lr}\text { Oral medications } & 15(50)\end{array}$

$\begin{array}{ll}\text { Metformin } & 14(47)\end{array}$

$\begin{array}{ll}\text { Sulfonylureas } & 4(13)\end{array}$

DPP-4 $1(3)$

$\begin{array}{ll}\text { GLP-1 agonist } & 0(0)\end{array}$

$\begin{array}{ll}\text { Pioglitazone } & 0(0)\end{array}$

SGLT2 inhibitor $1(3)$

$\begin{array}{lc}\text { Alpha-glucosidase inhibitor } & 6(20)\end{array}$

Insulin only $4(13)$

$\begin{array}{ll}\text { Short-acting } & 4(13)\end{array}$

Long-acting 


\section{Cureus}

\begin{tabular}{|c|c|}
\hline Oral medications and insulin therapy & $3(10)$ \\
\hline Other medications & $6(20)$ \\
\hline Herbs / Supplements & $7(23)$ \\
\hline \multicolumn{2}{|l|}{ Adherence to prescribed regimen } \\
\hline Yes & $18(60)$ \\
\hline No & $12(40)$ \\
\hline \multicolumn{2}{|l|}{ Blood pressure medication } \\
\hline Thiazide & $5(17)$ \\
\hline Loop diuretic & $0(0)$ \\
\hline Calcium channel blocker & $7(23)$ \\
\hline ACE inhibitor & $7(23)$ \\
\hline Angiotensin receptor blocker & $3(10)$ \\
\hline Beta blocker & $4(13)$ \\
\hline None & $0(0)$ \\
\hline \multicolumn{2}{|l|}{ Statin use } \\
\hline Yes & $16(53)$ \\
\hline No & $14(47)$ \\
\hline \multicolumn{2}{|l|}{ Aspirin use } \\
\hline Yes & $10(33)$ \\
\hline No & $20(67)$ \\
\hline \multicolumn{2}{|l|}{ Barriers to diabetes care } \\
\hline Local healthcare provider availability & $5(17)$ \\
\hline Cost of healthcare provider visit & $10(33)$ \\
\hline Transportation to healthcare provider & $3(10)$ \\
\hline Availability of medications at pharmacy, hospital, health center, or mail & $4(13)$ \\
\hline Cost of medications & $10(33)$ \\
\hline Medication side effect concern & $8(27)$ \\
\hline Lack of education on diabetes treatment & $3(10)$ \\
\hline None & $8(27)$ \\
\hline \multicolumn{2}{|l|}{ BMI } \\
\hline Mean (SD) & $26.6(8.5)$ \\
\hline \multicolumn{2}{|l|}{ Blood glucose } \\
\hline Median (range) & $140(95-407)$ \\
\hline \multicolumn{2}{|l|}{ HbA1C } \\
\hline Median (range) & $7.45(6-11.7)$ \\
\hline
\end{tabular}

TABLE 1: Socioeconomic, anthropometric, and diabetes care-related data for adults participating in free diabetes self-management classes 


\section{Cureus}

The mean score in the NLit was 38.1 ( $\left(D^{ \pm 9} 9\right.$.4), which corresponds with a likelihood of poor nutrition literacy. The maximum score was 56 , which corresponds with the possibility of poor nutrition literacy. There were no patients who scored in the range of likelihood of good nutrition literacy (Table 2). Gender, race, and education did not significantly impact NLit score.

Characteristic

Mean NLit score (SD)

Sex

NLit score

Male sex

Female sex

p-value

Race

NLit score

Middle Eastern

Latino / Hispanic

Black

White

Asian-Indian Subcontinental

Other

p-value

Education level

NLit score

None

35

Elementary

37

Middle school

39

High school

39.3

College degree

38.4

Graduate school

36.5

Post-graduate

p-value
39.4

45

Participants $(n=30)$

38.1 (9.4)

35.9

40.3

0.204

40.5

37.5

30.4

35

0.397

TABLE 2: Mean Nutrition Literacy Assessment score based on sex, ethnicity, and education level for adults participating in free diabetes self-management classes

Patients scored the lowest in the domain of food numeracy and labels (Mean score: 4.2 SD \pm 2.2 ) and scored the highest in food groups (mean score $11.7 \mathrm{SD}^{ \pm} 3.6$ ), compared to all other sections (Table 3). 


\section{Cureus}

Domain Type

Nutrition and health

Number of questions

Mean correct (SD)

Energy Sources in Food

Number of questions

Mean correct (SD)

Household food measurement

Number of questions

Mean correct (SD)

Food label and numeracy

Number of questions

Mean correct (SD)

Food groups

Number of questions

16

Mean correct (SD)

Consumer skills

Number of questions

Mean correct (SD)

10

3)

Participants $(n=30)$ 


\section{Discussion}

There is a direct correlation between medical nutritional therapy and diabetes control [19]. The American Diabetes Association recommends that all patients with diabetes receive individualized medical nutrition therapy to promote healthy eating habits and improve HbA1c, blood pressure, and cholesterol levels. Nevertheless, it is essential to note there are intricate complexities to this task since dietary modification is dependent on many factors. These include health literacy, cultural preferences, personal preferences, access to healthy food choices, and willingness to change lifestyle [20]. Several scholars have proposed that nutrition literacy is a domain of health literacy [21].

Low health literacy has been described as a barrier to improving health outcomes in groups with ethnic or racial disparities [7]. Although low education has been associated with low health literacy [22,23], there was no significant difference in nutrition literacy based on education level or ethnicity in our cohort. Interestingly, none of the subjects scored in the highest category, the likelihood of good nutrition literacy. Most of them ( $n=22,73 \%)$ scored in the lowest category, the likelihood of poor nutrition literacy. Only $10 \%$ of the subjects who participated recognized that lack of education regarding diabetes treatment was a barrier to diabetes care.

Our results also showed that the subjects who scored in the likelihood of poor nutrition literacy category had an average higher $\mathrm{HbA1c}$ than those who scored in the possibility of poor nutrition literacy. These results were statistically significant, with a mean difference in HbA1c of $1.9 \%$ ( CI- 0.6-3.0, p=0.005). Poor nutrition literacy could be a barrier to diabetes control. Assessment of nutrition literacy before the start of medical nutrition therapy and after could be helpful in understanding and guiding patients to modify their diet and achieve treatment goals optimally.

Furthermore, this difference in HbA1c, which we would expect to be even more if comparing patients in the likelihood of poor nutrition literacy to those in the range of good nutrition literacy, could potentially have a significant clinical impact on health outcomes and healthcare expenditure. It is essential to highlight that currently available medications for the treatment of diabetes decrease HbA1c approximately by $0.5-1.5 \%$, and based on our results, medical nutrition therapy could be of a higher impact than medications [24].

The NLit assessment tested six nutrition domains. Subjects scored the worst in the Food numeracy and Labels. Data regarding health literacy, food label interpretation, and food numeracy is controversial. Some studies have shown that patients with limited health literacy have greater difficulty interpreting food labels and are less likely to refer to food labels [25-27]. However, other studies have shown a negative association between health literacy and food labels [28-30]. Nevertheless, focused didactics on food numeracy and label instruction may be a high yield topic during medical nutrition therapy based on our results.

There are several limitations to this study. Some diabetes-related data was self-reported due to a lack of medical records access. Monthly resources and advertising limited our class size. None of our subjects scored in the likelihood of good nutrition literacy category, and we could not assess if there were a significant difference in HbA1c in this subgroup. Longitudinal studies and post-intervention analyses were not possible due to a lack of consistent monthly follow-up. Future studies with a larger sample size may be beneficial to elucidate further understanding of nutrition literacy and how this can improve diabetes outcomes in groups with socioeconomic disparities.

\section{Conclusions}

To our knowledge, this is the first study utilizing the Nutrition Literacy (NLit) assessment to evaluate nutrition literacy in uninsured subjects with diabetes mellitus. This study demonstrated that poor nutrition literacy is associated with worse glycemic control among uninsured patients with diabetes mellitus based on the Nutrition Literacy Assessment. We suggest a possible role in assessing nutrition literacy in patients with diabetes to identify deficiencies that could be improved upon with adequate medical, nutritional therapy. Furthermore, empowering patients with diabetes and providing broader access to nutritional education to improve nutrition literacy could represent a high yield intervention that can improve health outcomes and reduce the cost associated with the disease. More research is needed to understand better how nutritional literacy optimizes or modulates glycemic control among uninsured and insured patients to address health outcomes and disparities.

\section{Additional Information \\ Disclosures}

Human subjects: Consent was obtained or waived by all participants in this study. University of South Florida Research and Innovation issued approval Pro00023920. 12/21/2015 Sayeef Mirza Global Health 18012 cozumel isle drive Tampa, FL 33647 RE: Expedited Approval for Initial Review IRB\#: Pro00023920 Title: Assessing Burden of Chronic Disease (ABCD) among the Uninsured of Tampa Bay Study Approval Period: 12/21/2015 to 12/21/2016 Dear Sayeef Mirza: On12/21/2015, the Institutional Review Board (IRB) reviewed and APPROVED the above application and all documents contained within, including those outlined below. 
Approved Item(s): Protocol Document(s): Protocol It was the determination of the IRB that your study qualified for expedited review which includes activities that (1) present no more than minimal risk to human subjects, and (2) involve only procedures listed in one or more of the categories outlined below. The IRB may review research through the expedited review procedure authorized by 45CFR46.110 and 21 CFR 56.110. The research proposed in this study is categorized under the following expedited review category: (5) Research involving materials (data, documents, records, or specimens) that have been collected, or will be collected solely for nonresearch purposes (such as medical treatment or diagnosis). Your study qualifies for a waiver of the requirements for the informed consent process as outlined in the federal regulations at 45CFR46.116 (d) which states that an IRB may approve a consent procedure which does not include, or which alters, some or all of the elements of informed consent, or waive the requirements to obtain informed consent provided the IRB finds and documents that (1) the research involves no more than minimal risk to the subjects; (2) the waiver or alteration will not adversely affect the rights and welfare of the subjects; (3) the research could not practicably be carried out without the waiver or alteration; and (4) whenever appropriate, the subjects will be provided with additional pertinent information after participation. Your study qualifies for a waiver of the requirement for signed authorization as outlined in the HIPAA Privacy Rule regulations at 45CFR164.512(i) which states that an IRB may approve a waiver or alteration of the authorization requirement provided that the following criteria are met (1) the PHI use or disclosure involves no more than a minimal risk to the privacy of individuals; (2) the research could not practicably be conducted without the requested waiver or alteration; and (3) the research could not practicably be conducted without access to and use of the PHI. A waiver of HIPAA Authorization is granted for this retrospective chart review of patients seen between September 30, 2014 and October 1, 2015 in one of the following five free clinics in the Tampa Bay Area: BRIDGE Clinic, Judeo Christian Health Clinic, Red Crescent Clinic of Tampa Bay, Brandon Outreach Clinic, The Well. This waiver allows the study team to obtain PHI of patients in this cohort from the medical records at each of these clinics (paper or electronic). As the principal investigator of this study, it is your responsibility to conduct this study in accordance with IRB policies and procedures and as approved by the IRB. Any changes to the approved research must be submitted to the IRB for review and approval via an amendment. Additionally, all unanticipated problems must be reported to the USF IRB within five (5) calendar days. We appreciate your dedication to the ethical conduct of human subject research at the University of South Florida and your continued commitment to human research protections. If you have any questions regarding this matter, please call 813-974-5638. Sincerely, E. Verena Jorgensen, M.D., Chairperson USF Institutional Review Board. Animal subjects: All authors have confirmed that this study did not involve animal subjects or tissue. Conflicts of interest: In compliance with the ICMJE uniform disclosure form, all authors declare the following: Payment/services info: All authors have declared that no financial support was received from any organization for the submitted work. Financial relationships: All authors have declared that they have no financial relationships at present or within the previous three years with any organizations that might have an interest in the submitted work. Other relationships: All authors have declared that there are no other relationships or activities that could appear to have influenced the submitted work.

\section{Acknowledgements}

Funding provided by University of South Florida, Graduate Medical Education Grant The authors confirm contribution to the paper as follows: study conception and design: B.Rivera Rivero, A-S Mirza, A.Makarova, D. Sidig, S. Niazi, N. Weare-Regales; data collection: B. Rivera Rivero, A.Makarova, R. Abddelgader, S.Mirza, H. Joud, M.Urfi, O. Jureyda, D.Sidig, S.Niazi, A.Ahmed ; analysis and interpretation of results: B. Rivera Rivero, A-S. Mirza, N.Weare-Regales, A.Makarova, J. Swanson; draft manuscript preparation: B. Rivera Rivero, A-S Mirza, N.Weare-Regales, A. Makarova, J.Swanson, F.Khan, M.Siddique. All authors reviewed the results, draft manuscript, and approved the final version of the manuscript.

\section{References}

1. Centers for Disease Control and Prevention. National Diabetes Statistics Report, 2020. Atlanta, GA: Centers for Disease Control and Prevention, U.S. Dept of Health and Human Services. (2020). Accessed: November 5, 2020: https://www.cdc.gov/diabetes/pdfs/data/statistics/national-diabetes-statistics-report.pdf.

2. American Diabetes A: Economic costs of diabetes in the U.S. in 2017 . Diabetes Care. 2018, 41:917-28. 10.2337/dci18-0007

3. Nelson KM, Chapko MK, Reiber G, Boyko EJ: The association between health insurance coverage and diabetes care; data from the 2000 Behavioral Risk Factor Surveillance System. Health Serv Res. 2005, 40:361-72. 10.1111/j.1475-6773.2005.00361.x

4. Rahman S, Mirza AS, Wathington D, Green S, Mayers Y, Iranmanesh E, Woodard L: Chronic disease and socioeconomic factors among uninsured patients: A retrospective study. Chronic Illn. 2021, 17:53-66. 10.1177/1742395319828430

5. Madden MH, Tomsik P, Terchek J, et al.: Keys to successful diabetes self-management for uninsured patients: social support, observational learning, and turning points: a safety net providers' strategic alliance study. J Natl Med Assoc. 2011, 103:257-64. 10.1016/s0027-9684(15)30292-3

6. Hersh L, Salzman B, Snyderman D: Health literacy in primary care practice . Am Fam Physician. 2015, 92:118-24.

7. Tseng HM, Liao SF, Wen YP, Chuang YJ: Stages of change concept of the transtheoretical model for healthy eating links health literacy and diabetes knowledge to glycemic control in people with type 2 diabetes. Prim Care Diabetes. 2017, 11:29-36. 10.1016/j.pcd.2016.08.005

8. Fransen MP, von Wagner C, Essink-Bot ML: Diabetes self-management in patients with low health literacy: 
ordering findings from literature in a health literacy framework. Patient Educ Couns. 2012, 88:44-53. 10.1016/j.pec.2011.11.015

9. Al Sayah F, Majumdar SR, Williams B, Robertson S, Johnson JA: Health literacy and health outcomes in diabetes: a systematic review. J Gen Intern Med. 2013, 28:444-52. 10.1007/s11606-012-2241-z

10. Eichler K, Wieser S, Brügger U: The costs of limited health literacy: a systematic review . Int J Public Health. 2009, 54:313-24. 10.1007/s00038-009-0058-2

11. Ahola AJ, Groop PH: Barriers to self-management of diabetes . Diabet Med. 2013, 30:413-20. 10.1111/dme.12105

12. Kutner M, Greenberg E, Jin Y, Paulsen C: The health literacy of America's adults: results from the 2003 national assessment of adult literacy. NCES. 2006, 483:

13. Brega AG, Barnard J, Mabachi NM, et al.: AHRQ Health Literacy Universal Precautions Toolkit, Second Edition. University of Colorado Anschutz Medical Campus, Rockville, MD; 2015.

14. Carbone ET, Gibbs HD: Measuring nutrition literacy: problems and potential solutions . J Nutr Disorders Ther. 2013 , 3:e105. 10.4172/2161-0509.1000e105

15. Lino M, Basiotis PP, Anand RS, Variyam JN: The diet quality of Americans: strong link with nutrition knowledge. Family Economics and Nutrition Review. 1999, 12:49.

16. Gibbs HD, Ellerbeck EF, Gajewski B, Zhang C, Sullivan DK: The nutrition literacy assessment instrument is a valid and reliable measure of nutrition literacy in adults with chronic disease. J Nutr Educ Behav. 2018, 50:247-257. 10.1016/j.jneb.2017.10.008

17. Harris PA, Taylor R, Thielke R, Payne J, Gonzalez N, Conde JG: Research electronic data capture (REDCap)-a metadata-driven methodology and workflow process for providing translational research informatics support. J Biomed Inform. 2009, 42:377-81. 10.1016/j.jbi.2008.08.010

18. Rahman S, Mirza AS, Stenback J, et al.: Burden of chronic conditions among patients from free clinics: a retrospective chart review of 2015. J Health Care Poor Underserved. 2018, 29:1011-26. 10.1353/hpu.2018.0076

19. Morris SF, Wylie-Rosett J: Medical nutrition therapy: a key to diabetes management and prevention . Clin Diabetes. 2010, 28:12-18. 10.2337/diaclin.28.1.12

20. Evert AB, Dennison M, Gardner CD, et al.: Nutrition therapy for adults with diabetes or prediabetes: a consensus report. Diabetes Care. 2019, 42:731-54. 10.2337/dci19-0014

21. Velardo S: The nuances of health literacy, nutrition literacy, and food literacy . J Nutr Educ Behav. 2015, 47:385-9. 10.1016/j.jneb.2015.04.328

22. Van der Heide I, Wang J, Droomers M, Spreeuwenberg P, Rademakers J, Uiters E: The relationship between health, education, and health literacy: results from the Dutch adult literacy and life skills survey. J Health Commun. 2013, 18:172-84. 10.1080/10810730.2013.825668

23. Rudd RE: Health literacy skills of U.S. adults . Am J Health Behav. 2007, 31:s8-18. 10.5555/ajhb.2007.31.supp.S8

24. Sherifali D, Nerenberg K, Pullenayegum E, Cheng JE, Gerstein HC: The effect of oral antidiabetic agents on A1C levels: a systematic review and meta-analysis. Diabetes Care. 2010, 33:1859-64. 10.2337/dc09-1727

25. Cha E, Kim KH, Lerner HM, Dawkins CR, Bello MK, Umpierrez G, Dunbar SB: Health literacy, self-efficacy, food label use, and diet in young adults. Am J Health Behav. 2014, 38:331-9. 10.5993/AJHB.38.3.2

26. Miller LM, Cassady DL: The effects of nutrition knowledge on food label use. a review of the literature . Appetite. 2015, 92:207-16. 10.1016/j.appet.2015.05.029

27. Sinclair S, Hammond D, Goodman S: Sociodemographic differences in the comprehension of nutritional labels on food products. J Nutr Educ Behav. 2013, 45:767-72. 10.1016/j.jneb.2013.04.262

28. Vijaykumar S, Lwin MO, Chao J, Au C: Determinants of food label use among supermarket shoppers: a Singaporean perspective. J Nutr Educ Behav. 2013, 45:204-12. 10.1016/j.jneb.2012.09.001

29. Speirs KE, Messina LA, Munger AL, Grutzmacher SK: Health literacy and nutrition behaviors among lowincome adults. J Health Care Poor Underserved. 2012, 23:1082-91. 10.1353/hpu.2012.0113

30. Malloy-Weir L, Cooper M: Health literacy, literacy, numeracy and nutrition label understanding and use: a scoping review of the literature. J Hum Nutr Diet. 2017, 30:309-25. 10.1111/jhn.12428 